\title{
Improved spatial resolution for reflection mode infrared microscopy
}

\author{
Hans A. Bechtel, ${ }^{1}$ Michael C. Martin, ${ }^{1}{ }^{1, a)}$ T. E. May, ${ }^{2}$ and Philippe Lerch ${ }^{3}$ \\ ${ }^{1}$ Advanced Light Source Division, Lawrence Berkeley National Laboratory, 1 Cyclotron Road, Berkeley, \\ California 94720, USA \\ ${ }^{2}$ Canadian Light Source, Inc., University of Saskatchewan, 101 Perimeter Road, Saskatoon, Saskatchewan \\ S7N 0X4, Canada \\ ${ }^{3}$ Swiss Light Source, Paul Scherrer Institut, 5232 Villigen, Switzerland
}

(Received 6 October 2009; accepted 7 November 2009; published online 16 December 2009)

\begin{abstract}
Standard commercial infrared microscopes operating in reflection mode use a mirror to direct the reflected light from the sample to the detector. This mirror blocks about half of the incident light, however, and thus degrades the spatial resolution by reducing the numerical aperture of the objective. Here, we replace the mirror with a $50 \%$ beamsplitter to allow full illumination of the objective and retain a way to direct the reflected light to the detector. The improved spatial resolution is demonstrated using two different microscopes capable of diffraction-limited resolution: the first microscope is coupled to a synchrotron source and utilizes a single point detector, whereas the second microscope has a standard blackbody source and uses a focal plane array detector.

(C) 2009 American Institute of Physics. [doi:10.1063/1.3270260]
\end{abstract}

Infrared (IR) microscopes are typically designed to be used with standard blackbody sources. Because these sources suffer from poor brightness, spatial resolution is controlled by geometrical apertures that are typically limited by signalto-noise ratios to $20 \mu \mathrm{m}$ or larger. In the mid-IR, these aperture sizes are far from the diffraction limit. Consequently, most designers choose to maximize the throughput of the system rather than achieve the best possible spatial resolution at the diffraction limit. Technological advances in IR sources and detectors, namely, synchrotron IR radiation ${ }^{1-9}$ and focal plane arrays; ${ }^{10}$ however, have made diffractionlimited spatial resolutions possible by either increasing the source brightness ${ }^{11}$ or by multiplexing data acquisition. Here, we discuss a simple modification to commercial IR microscopes that allows the user to take full advantage of the spatial resolution achievable by these new sources and detectors.

IR microscopes operate in either transmission or reflection mode, with the choice primarily depending on the optical properties of the sample and/or the substrate. In transmission mode [Fig. 1(a)], an objective focuses the IR radiation onto the sample and a second objective or condenser collects the transmitted radiation before directing it to the detector. In the standard reflection mode [Fig. 1(b)], light that is reflected off the sample is collected by the same objective that focuses the IR light. The light is then directed to the detector by a "sluice" mirror, which is removed during transmission mode measurements. The mirror, however, blocks a portion of the incident light and effectively shadows half of the objective's secondary mirror. Because the spot size for a diffractionlimited system is determined by the wavelength of light and the numerical aperture (NA) of the objective, this halfillumination degrades the spatial resolution of the microscope.

\footnotetext{
${ }^{\text {a) }}$ Author to whom correspondence should be addressed. Electronic mail: mcmartin@lbl.gov. Tel.: 510-495-2231. FAX: 510-495-2067.
}

The spot size in a diffraction-limited system can be modeled by the point spread function (PSF) of the objective. Figures 2(a)-2(c) show the simulated PSF (Ref. 12) of a common Schwarzschild objective used in mid-IR microscopy $(32 \times$, NA 0.65$)$ with full illumination of the secondary mirror, as is the case with transmission mode. The PSF of a Schwarzschild objective has a narrower zero-order peak and larger first-order and higher order diffraction maxima than the familiar Airy pattern of a typical visible microscope objective because the secondary mirror of the Schwarzschild objective obscures a portion of the central aperture. While a standard optic has about $85 \%$ of the total sensitivity in the zero-order peak, the Schwarzschild objective has only $50 \%$ in the central region, ${ }^{4}$ leading to possible loss of image contrast when used for scanning microscopy. The effects of the first-order and higher order diffraction maxima on resolving power, however, can be reduced by operating the microscope in a confocal arrangement, as demonstrated by Carr. ${ }^{4}$

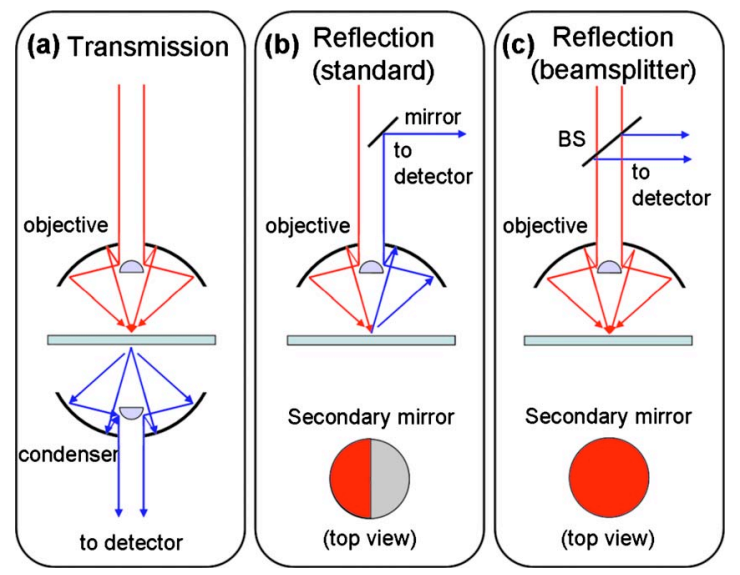

FIG. 1. (Color online) Schematic diagram of three different modes of IR microscopy: (a) transmission, (b) reflection with the standard mirror design, and (c) reflection with the beamsplitter design. 

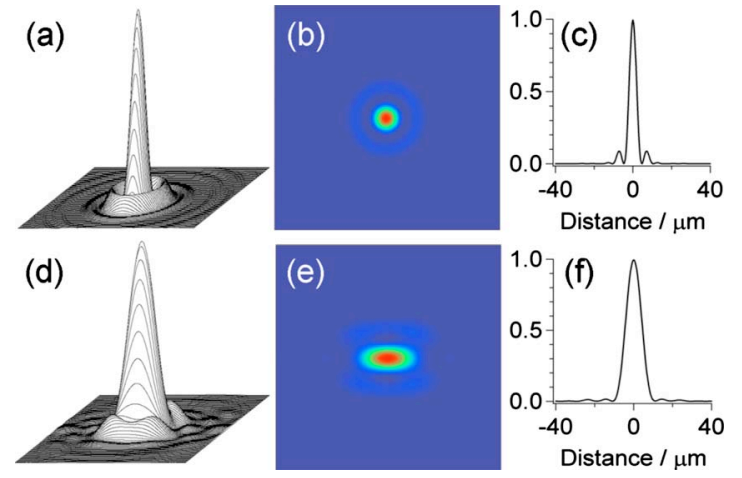

FIG. 2. (Color online) (a) Surface plot, (b) image plot, and (c) horizontal cross section of the PSF of a $32 \times$ Schwarzschild objective with full illumination of the secondary mirror at $\lambda=6 \mu \mathrm{m}$, which is the case for the transmission and beamsplitter reflection modes. (d) Surface plot, (e) image plot, and (f) horizontal cross section of the PSF of the same objective with halfillumination of the secondary mirror, which is the case for the standard reflection mode.

Figures 2(d)-2(f) show the PSF of the same $32 \times$ Schwarzschild objective when the secondary mirror is halfilluminated, as is the case with the standard reflection mode. The reduction in NA creates a spot size that is more than double in one dimension than in the other dimension. This elliptical spot can distort images created by scanning microscopy, particularly at longer wavelengths where the effects of diffraction are more obvious. An alternative method of reflection that achieves the same resolution as in transmission

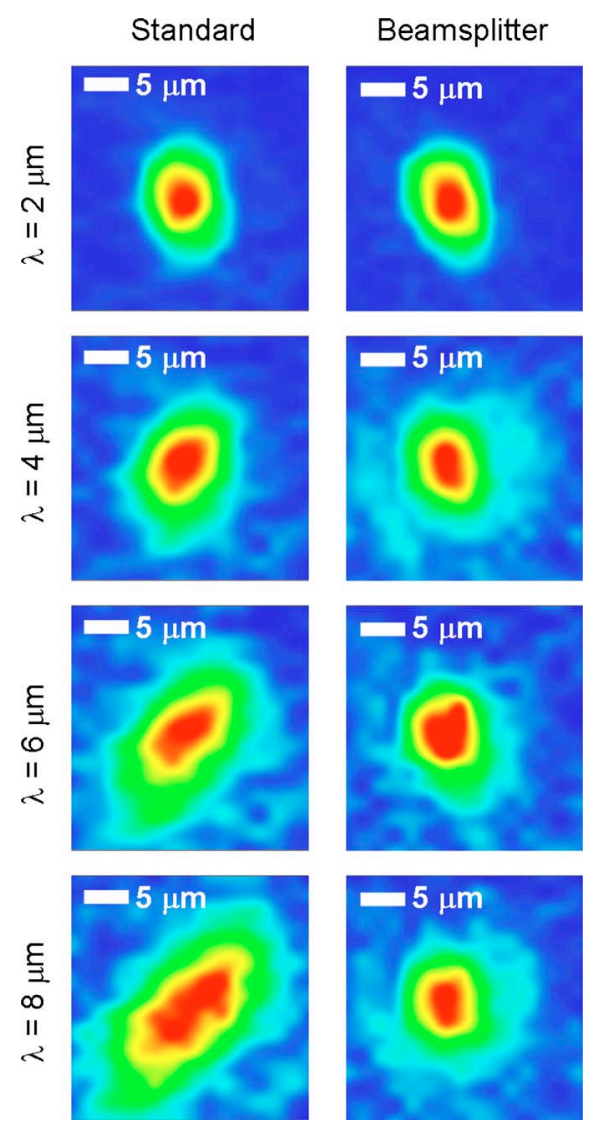

FIG. 3. (Color online) Images of an $8 \mu \mathrm{m} \mathrm{Ti} \mathrm{dot} \mathrm{on} \mathrm{a} \mathrm{Si}$ substrate at different wavelengths for standard and beamsplitter reflection modes using a synchrotron source and a single point detector.

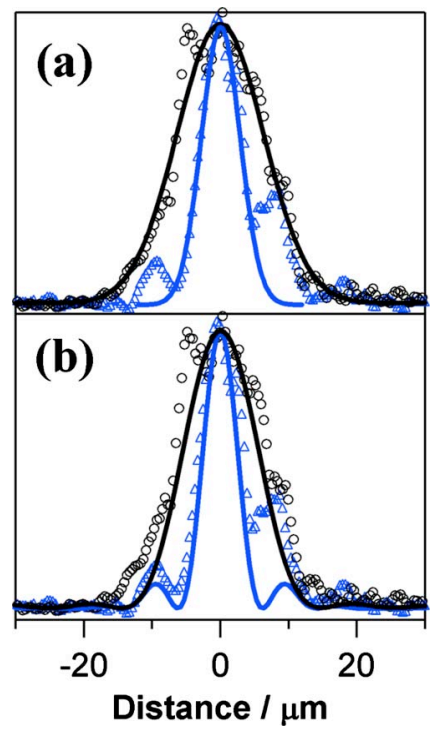

FIG. 4. (Color online) (a) Gaussian fits and (b) PSF simulations (solid lines) superimposed on the first derivatives of step edge profiles at $\lambda=8 \mu \mathrm{m}$ for the standard reflection mode (circles) and the beamsplitter reflection mode (triangles) using a synchrotron source and a single point detector.

mode replaces the sluice mirror with a beamsplitter [Fig. $1(\mathrm{c})$ ]. Although this design limits the throughput to $50 \%$ of the standard reflection mode, the maximal spatial resolution for the microscope is achieved because the secondary mirror is fully illuminated.

In order to experimentally verify the improved spatial resolution of the beamsplitter reflection mode, IR measurements were performed at the Advanced Light Source (ALS) and the Swiss Light Source (SLS). For measurements at the ALS, synchrotron IR light at beamline 1.4.3 was directed through a Nicolet Magna 760 Fourier transform infrared (FTIR) interferometer bench with a $\mathrm{KBr}$ beamsplitter and a Spectra Tech Nic-Plan IR microscope $(32 \times$ objective, NA $=0.65$ ) before being detected with a mercury cadmium telluride (MCT-A) detector. Sample images were acquired with an automated microscope stage (Prior ProScan II) capable of step sizes as small as $0.1 \mu \mathrm{m}$. For reflection measurements in beamsplitter mode, the Nic-Plan microscope was modified to accommodate a 50:50 $\mathrm{CaF}_{2}$ beamsplitter (ISP Optics) at the position of the sluice mirror. In this case, the $\mathrm{CaF}_{2}$ beamsplitter limits the spectral range to wavelengths shorter than $10 \mu \mathrm{m}$, but other beamsplitter substrates (e.g., $\mathrm{KBr}$ ) could be used to obtain spectra of the entire mid-IR spectral region if necessary. The modifications to the microscope allow easy switching between the standard and beamsplitter reflection modes. For measurements at the SLS, IR light from a globar source was directed through a Bruker Vertex 70 FTIR interferometer with a $\mathrm{KBr}$ beamsplitter and coupled into a Bruker Hyperion 3000 microscope with a 128 element FPA detector. For reflection measurements in beamsplitter mode, the sluice mirror was replaced with a metal mesh that acted as a beamsplitter in the mid-IR spectral region.

Three types of resolution tests were performed in order to demonstrate the improved spatial resolution of the beamsplitter reflection mode. In the first test, an $8 \mu \mathrm{m}$ Ti dot on a Si substrate was imaged using the standard and beamsplitter reflection modes (Fig. 3) with a synchrotron source. At $\lambda$ $=2 \mu \mathrm{m}$, the two images are nearly identical because the dif- 

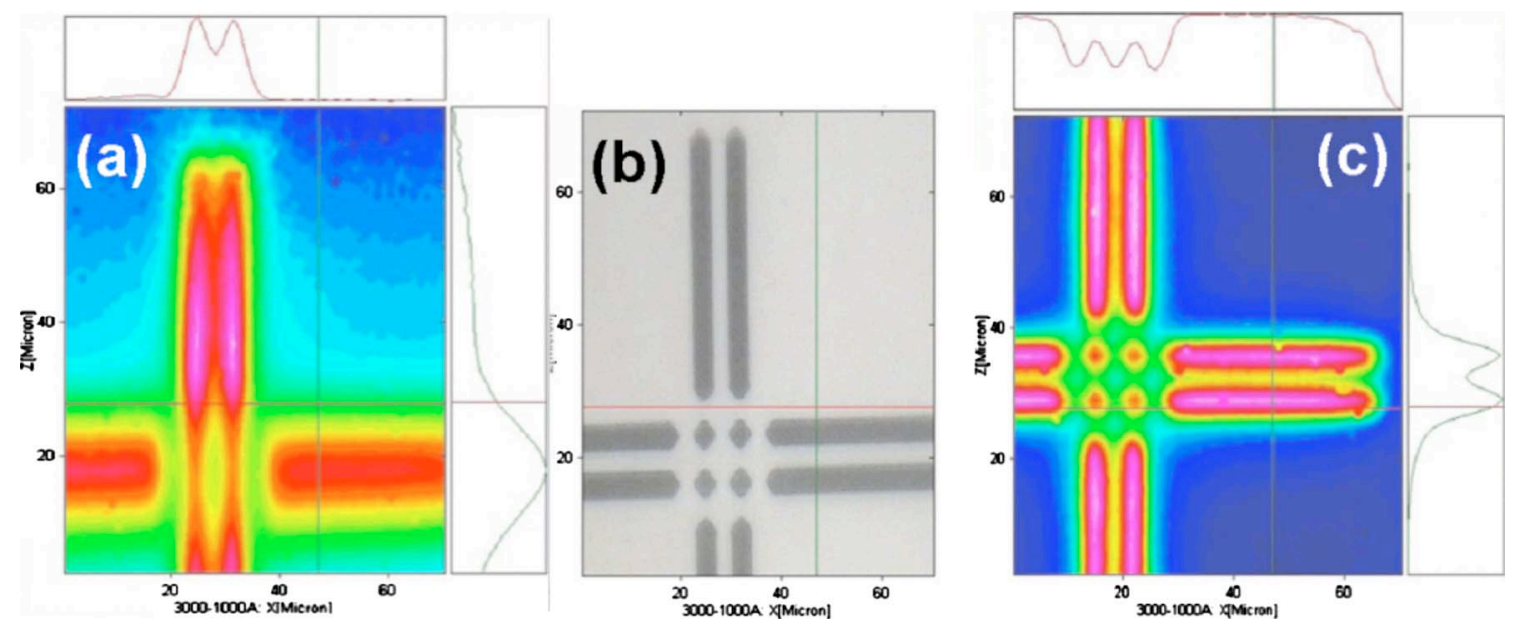

FIG. 5. (Color online) IR images of a patterned sample obtained with (a) standard reflection mode and (c) beamsplitter reflection mode using a globar source and a focal plane array. (b) Optical image of the sample.

fraction pattern for both modes of reflection is smaller than the test sample. At longer wavelengths, however, the standard reflection mode images are clearly distorted in one dimension and appear elliptical (oriented at approximately $45^{\circ}$ because of the optical design of the microscope). The images from the beamsplitter reflection mode, on the other hand, remain symmetrical and relatively unchanged with increasing wavelength, in agreement with the PSF simulations.

The second resolution test was a step-edge (or knifeedge) test with a synchrotron source using a high resolution USAF 1951 3-Bar Resolving Test Chart (MILSTD-150A, Sec. 5.1.1.7) from Applied Image, Inc. (Rochester, NY, USA). The sample was stepped in $0.5 \mu \mathrm{m}$ increments from a position on the absorbing/transmitting glass pattern to a position on the reflective metal coating along a line oriented at $45^{\circ}$, corresponding to the degraded resolution in the standard reflection mode. By acquiring a spectrum at each point, a profile of the reflectivity as a function of position and wavelength was obtained. The first derivative of the profile and the FWHM of a Gaussian fit are comparable to the PSF and resolution of the system, respectively. ${ }^{13}$ Figure 4 shows the first derivative of the step profiles for the standard and beamsplitter reflection modes at $\lambda=8 \mu \mathrm{m}$ obtained without any apertures. The standard reflection mode profile is clearly wider than the zero and first-order diffraction maxima of the beamsplitter reflection mode profile. A simple linear fit to the data between $\lambda=2$ and $\lambda=8 \mu \mathrm{m}$ yields $(0.73 \pm 0.05) \lambda$ resolution for the beamsplitter reflection mode and $(1.83 \pm 0.05) \lambda$ resolution for the standard reflection mode.

The first two tests demonstrate the improved diffractionlimited performance of the beamsplitter reflection mode when using a synchrotron source, which is 100-1000 times brighter than a globar source. Indeed, the ability to achieve diffraction-limited performance with high signal-to-noise ratios is the primary reason that IR spectromicroscopy beamlines exist worldwide. Recent technological advances in mid-IR focal plane arrays, however, have enabled diffraction limited performance with globar sources, although synchrotron sources still retain their superiority in terms of ultimate spatial resolution and signal-to-noise ratios. ${ }^{14}$ Therefore, in the final resolution test, the beamsplitter reflection mode is examined with a microscope equipped with a globar source and a focal plane array detector. As seen in Fig. 5, the beam- splitter reflection mode provides superior resolving power over the standard reflection mode.

By illuminating the entire secondary mirror, the beamsplitter reflection mode achieves the same symmetric illumination and therefore the same diffraction-limited spatial resolution obtained in transmission mode. This improved spatial resolution over the standard reflection mode, however, comes at the expense of IR light throughput. If the measurements are shot-noise limited, the factor of two reduction in throughput should reduce the signal-to-noise ratio by $\sqrt{ } 2$. In measurements at the ALS, we find the signal-to-noise ratio of the beamsplitter reflection mode to be only slightly worse than the standard reflection mode because shot-noise is usually not the limiting noise source. An rms noise value of $<0.05 \%$ is routinely achieved on a $100 \%$ line on gold (128 scans, $4 \mathrm{~cm}^{-1}$ resolution) for the beamsplitter mode, allowing both good sensitivity and high spatial resolution for IR measurements of small samples or regions of samples.

The authors thank G. L. Carr for help with Zemax simulations. The Advanced Light Source is supported by the Director, Office of Science, Office of Basic Energy Sciences, of the U.S. Department of Energy under Contract No. DEAC02-05CH11231.

${ }^{1}$ J. A. Reffner, P. A. Martoglio, and G. P. Williams, Rev. Sci. Instrum. 66, 1298 (1995).

${ }^{2}$ G. L. Carr, J. A. Reffner, and G. P. Williams, Rev. Sci. Instrum. 66, 1490 (1995).

${ }^{3}$ M. C. Martin and W. R. McKinney, Mater. Res. Soc. Symp. Proc. 524, 11 (1998).

${ }^{4}$ G. L. Carr, Rev. Sci. Instrum. 72, 1613 (2001).

${ }^{5}$ M. C. Martin and W. R. McKinney, Ferroelectrics 249, 1 (2001).

${ }^{6}$ P. Dumas and M. J. Tobin, Spectroscopy Europe 15, 17 (2003).

${ }^{7}$ L. M. Miller and P. Dumas, Biochim. Biophys. Acta 1758, 846 (2006).

${ }^{8}$ E. Levenson, P. Lerch, and M. C. Martin, J. Synchrotron Radiat. 15, 323 (2008).

${ }^{9}$ E. Levenson, P. Lerch, and M. C. Martin, Infrared Phys. Technol. 51, 413 (2008).

${ }^{10}$ E. N. Lewis, P. J. Treado, R. C. Reeder, G. M. Story, A. E. Dowrey, C. Marcott, and I. W. Levin, Anal. Chem. 67, 3377 (1995).

${ }^{11}$ W. D. Duncan and G. P. Williams, Appl. Opt. 22, 2914 (1983).

${ }^{12}$ Zemax (Zemax Development Corporation).

${ }^{13}$ J. C. Russ, The Image Processing Handbook (CRS, Boca Raton, 2002).

${ }^{14}$ E. Levenson, P. Lerch, and M. C. Martin, Infrared Phys. Technol. 49, 45 (2006). 